\title{
Online exercise ECG signal orthogonalization
}

\section{Conference Paper · October 1996}

DOI: 10.1109/CIC.1996.542517 · Source: IEEE Xplore

\section{CITATIONS}

27

\section{2 authors:}

\section{Bilal Acar}

University of Sussex

46 PUBLICATIONS 875 CITATIONS

SEE PROFILE
READS

23

Some of the authors of this publication are also working on these related projects:

Project $\quad$ Lecture Notes on Electroacoustic Transduction View project

Impacts of Pressurized Irrigation Technologies on Efficient Water Resources Uses in Semi-Arid

Project

Climate of Konya Basin of Turkey View project 


\title{
Online Exercise ECG Signal Orthogonalization
}

\author{
Burak Acar and Hayrettin Köymen \\ Bilkent University, Dept. of Electrical and Electronics Engineering, Ankara, Turkey
}

\begin{abstract}
In this paper an efficient method of making use of the redundancy in standard 12 lead ECG signals to eliminate noise is described. The method is based on orthogonalization via online Singular Value Decomposition (SVD). Its application as a filter to remove $E M G$ noise and baseline wander are explained. A comparative study of ST analysis results of original and processed exercise $E C G$ data is reported.
\end{abstract}

\section{Introduction}

Exercise electrocardiogram (ECG) is contaminated by EMG noise and baseline wander (BW). Storage of data constitutes a problem because this requires high storage capacity.

Several techniques were reported in the literature to enhance the exercise ECG signals [1]. All of them are based on some sort of composite beat formation. The main objective in these approaches is to disregard the outliner sample values and filter out the noise which is assumed to be stationary and uncorrelated with the signal. Averaging and median filtering are two basic techniques applied with this objective. However these assumptions are too restrictive and usually most of them are compulationally involved.

Our algorithm [2], on the other hand, is a recursive one which approximates Singular Value Decomposition (SVD) first proposed by Vanderschoot et.al. [3], to seperate fetal ECG from maternal ECG signals. This approach is employed in the presented work to remove redundancy by the orthogonalization of input signals. Thus all the information contained in the input signals is represented in a fewer number of output signals. Since these signals are orthogonal to each other, no redundancy exists in the output signal set.

\section{Basis Of The Algorithm}

The algorithm is based on the online approximation of SVD of a matrix, $\mathbf{M} \in \mathbf{R}^{n \times n}$. SVD of $\mathbf{M}$ is given in [4] as

$$
\begin{aligned}
\mathbf{U}^{T} \mathbf{M V} & =\mathbf{\Sigma} \\
\mathbf{U U}^{T}=\mathbf{U}^{T} \mathbf{U}=I_{p} & , \quad \mathbf{V} \mathbf{V}^{T}=I_{n} .
\end{aligned}
$$

from which we can establish the relation between SVD of $\mathbf{M}$ and eigenvalue decomposition of $\mathrm{MM}^{T}$ as

$$
\mathbf{\Sigma}^{2}=\mathbf{U}^{T} \mathrm{MM}^{T} \mathbf{U}
$$

The algorithm is given by

$$
\begin{aligned}
\mathbf{U}_{0}=\mathbf{I} & , \mathbf{C}_{0}=\mathbf{0} \\
\text { for } i & =1 \quad \text { to } n \\
\mathbf{s}_{i} & =\mathbf{U}_{i-1}^{T} \mathbf{m}_{i} \\
\mathbf{B}_{i} & =\alpha^{2} \mathbf{C}_{i-1}+\mathbf{s}_{i} \mathbf{s}_{i}^{T} \\
\mathbf{Q}_{i} & =f\left(\mathbf{B}_{i}\right) \\
\mathbf{C}_{i} & =\mathbf{Q}_{i}^{T} \mathbf{B}_{i} \mathbf{Q}_{i} \\
\mathbf{U}_{i} & =\mathbf{U}_{i-1} \mathbf{Q}_{i} \\
\hat{\mathbf{m}}_{i} & =\mathbf{U}_{i-1} \hat{\mathbf{s}}_{i}
\end{aligned}
$$

where

$$
\begin{array}{r}
r=\operatorname{rank}(\mathbf{M}) \\
\hat{\mathbf{s}}=\left[s_{1} \ldots s_{r} 0 \ldots 0\right] \\
\mathbf{U}=\left[\widetilde{\mathbf{U}}_{r} \tilde{\mathbf{U}}_{n}\right] .
\end{array}
$$

Zeros in $\hat{\mathbf{s}}$ are substituted for noise channels. $\widetilde{\mathbf{U}}_{r}$ corresponds to high singular values and spans the signal space. $\widetilde{\mathbf{U}}_{n}$ is its orthogonal complement and spans the noise space. $\mathbf{Q}_{i}$ is the Given's Rotation matrix that makes an off diagonal element of $\mathbf{B}_{i}$ zero [4]. The maximum off diagonal element of $\mathbf{B}_{i}$ is chosen to be made zero at each step in the above algorithm. $\mathbf{m}_{i}$ is the input signal vector at time instant $i . \mathrm{s}_{i}$ corresponds to the decomposed signal set at that instant. $\hat{\mathbf{m}}_{i}$ is the reconstructed signal set. Figure 1 shows the input and the decomposed signal sets. The first $r$ cllannels $(r=2$ in the example) are the projections of input channels onto the subspace spanned by $\widetilde{\mathrm{U}}_{1}$. (see Equation 14). These make up the signal space. The rest of the decomposed signals are the projections onto the noise space spanned by $\widetilde{\mathbf{U}}_{n}$. 


\section{Noise Elimination}

In the following describe our online noise detection algorithm developed and tested on real data.

In general, the algorithm is capable of mapping noise and ECG onto orthogonal subspaces, the signal space and the noise space as mentioned before. However in some cases shifts between the two subspaces can occur and noise can be seen in the signal space. The reason for this differs. It may be due to high amplitude noise, low amplitude long lasting noise, noise in the majority of input channels, and noise in an important input channel. Noise detection and elimination is done to avoid such interferences into the signal space.

Two methods are developed for this purpose. High amplitude noise causes $\mathbf{u}_{i}$ 's $(i \leq \operatorname{rank}(\mathbf{M}))$ which define the projection of the input signal set onto $s_{i}$, to rotate significantly. The direction of rotation is such that the component of $\mathbf{u}_{i}$ corresponding to the noisy input channel experiences the highest increase in absolute value. As a result $\mathbf{u}_{i}$ begins to map noise onto the signal space. 'To preserve orthogonality some ECG signal is also mapped onto the noise space. Setting a. rotation angle threshold for $\mathbf{u}_{i}$ 's that span the signal space, we let them rotate within some limits so as not to cause any interference between subspaces as explained above. Whenever this threshold is violated, the noisy input chamel is determined for the components of $\mathbf{u}_{i}$.

Low amplitude noise is directly mapped onto the noise space. However if the noise in the input lasts for a long time, the energy content of the output noise channel begins to increase considerably depending on the value of $\alpha$. After a while, the diagonal entry of $\mathbf{C}$ corresponding to that noise channel reaches a signifcant fraction of the one corresponding to the smallest signal channel. This causes a rotation of $\mathbf{u}_{i}$ such that the noise is projected onto the signal space. This is a similar result as in high amplitude noise. However, these rotations are slower and cannot be detected by observing the rotation angle of $\mathbf{u}_{i}$. Rather, the accumulation can be detected by observing the diagonal entries of $\mathbf{C}$. When it is detected, the component of $\mathbf{u}_{i}$, mapping that particular noise, which is the greatest in absolute value shows the noisy input channel.

Having determined the noisy input channel, the corresponding row and left most column of $\mathbf{U}$ are deleted together with the last row and column of $\mathrm{C}$. This corresponds to excluding the noisy input chamel from the input data set, decreasing the dimension by one, disregarding the smallest SVD output channel.

These input channels, excluded from the decomposition, are checked via cross correlation of QRS com-

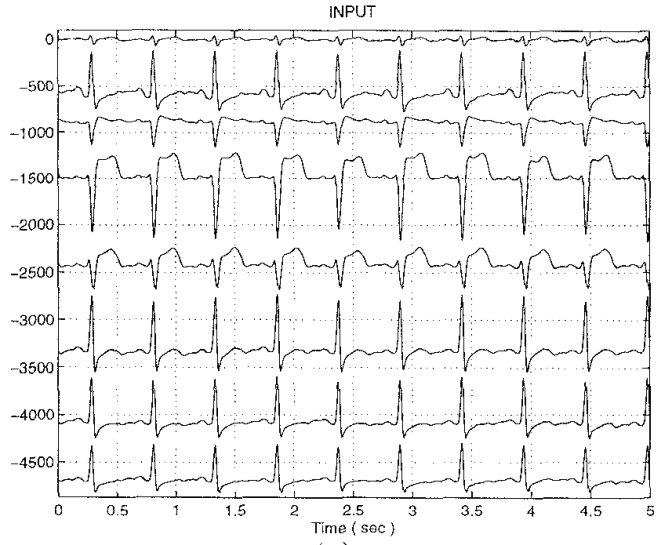

(a)

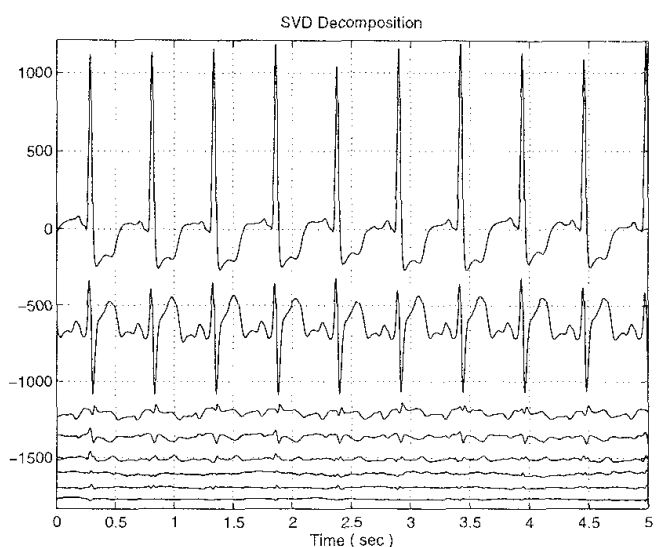

(b)

Figure 1: 8 channel (a)Input, (b)SVD Outputs

plexes to detect whether the noise has disappeared. The segments of the noisy input channel to be cross correlated are chosen from the clean input channels? QRS containing portions by time alignment. If a high correlation occurs then that channel is included in the input data set to be decomposed. This is done by increasing the dimension by recovering old $\mathbf{U}$ and $\mathbf{C}$ used just before the most recent dimension reduction.

\section{Observations}

Exhaustive analysis of exercise ECG data records from 23 patients showed that rank of $M$ is generally two and in few cases a third channel is seen in which considerable amount of QRS information exists.

High noise contamination of the signal channels were observed in case of noisy DII. This indicates that DII is the channel that is the least correlated channel with other input chamels. Hence its contribution cannot be 
substituted by signal content in other channels. This is due to its spatial position on the body. Recording DIII independently will help improving this unique dependence on DII and increase the noise immunity by adding further redundancy to the input set.

The forgetting factor, $\alpha$, determines how far the algorithm remembers past data. It affects the settling time and noise accumulation. As $\alpha$ gets higher, the probability of passing noise accumulation threshold falls, i.e. low power noise is mapped onto the noise space for longer periods. The decrease in the adaptive nature of the algorithm is a drawback for high $\alpha$. As $\alpha$ gets lower, immunity to noise is lost. If it is too low, ECG cannot be preserved in the signal space even in the absence of noise. Because the algorithm cannot remember past data at all and is extraordinarily sensitive to the input. As a result, QRS complexes cause high rotations of $\mathbf{u}_{i}$ 's much like high amplitude noise does. Good results were obtained with $\alpha=1-2^{-13}$.

The algorithm is sensitive to DC levels. DC components behave like independent signal sources. 'The rank of $\mathbf{M}$ increases to accomplish this. This means more QRS containing channels are seen at the output. 'They do not contain signals with different morphologies but the same signals with different DC levels. The only information they add to the decomposed signal set is the DC value. A first order Butterworth high pass filter with a cut-off frequency of $0.7 \mathrm{~Hz}$ is applied to the input signal to assure a zero DC level. Since the order of the filter is low, it does not affect the morphology of ECG.

The third signal channel, when exists, is a low am plitude channel. It is not immune to noise as much as other output channels. To overcome this problem, a limit is enforced on the speed of the rotation of $\mathbf{u}_{3}$.

It can be observed that the algorithm is capable of seperating EMG noise and BW, rpovided that the forgetting factor is chosen properly. 'The decomposed signal sets can be seen in Figure 2 .

\section{ST Analysis}

ST segment's potential level measurements were done both on the original and reconstructed data sets at two points, 60 and $80 \mathrm{~ms}$ after QRS. ECG records in Figures 3 and 4 , which are 14:40 min. and 21:40 min. long respectively, are sampled at 500 samples/sec. ST measurements were taken on average beats of successive $20 \mathrm{sec}$. episodes. Data in Figure 3 is taken from ECG with ST level variation and has 44 data points. Data in Figure 4 is taken from ECG without ST level variation and has 65 data points. The measurements were compared using linear regression analysis for each

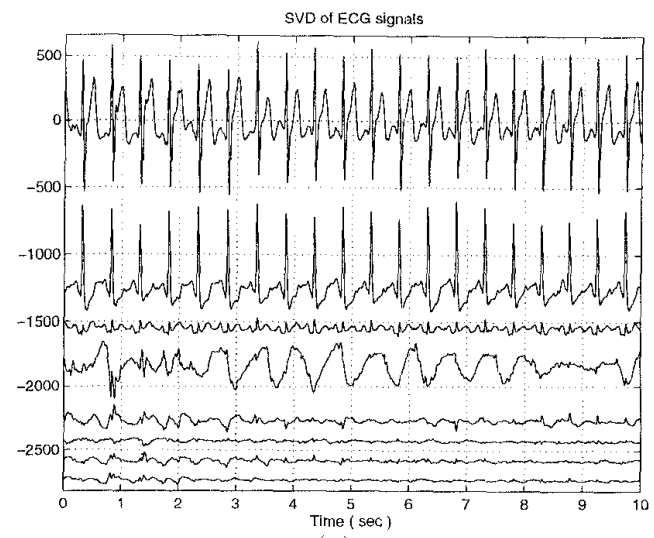

(a)

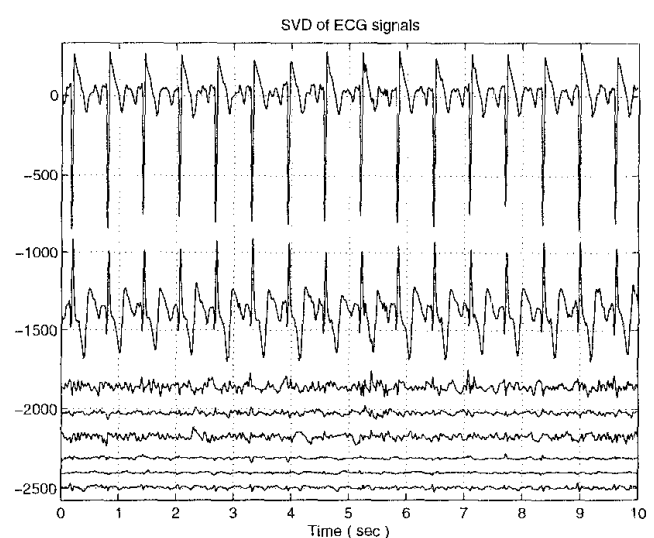

(b)

Figure 2: (a) SVD of ECG With $B W$ (b) SVD Of ECG With EMG

channel. The model used is $y_{i}=a \times x_{i}+b$, where $y_{i}$ 's and $x_{i}$ 's are ST measurements of original and reconstructed data, respectively. In the data with ST level shifts, a line with a slope close to 1 is fitted by MINITAB. ST measurements on channel DII, which is the hardest to reconstruct are compared in Figures 3 and 4 . The regression lines in Figure 3 and 4 are $y_{i}=0.991 \times x_{i}-0.028$ and $\left.y_{i}=0.913 \times x_{i}-0.018\right)$. In the data from normal ECG, which has almost constant ST levels, no line is fitted, however, the ST measurements in both data sets are almost equal (see Figure 4). The enhancement in ECG is clear when the number of QRS complexes included in the measurements are compared. For the data in Figure 3 the number of QRS complexes accepted in DII are 1625 and 1765 for original and reconstructed signals respectively. They are 1004 and 1058 for the data in Figure 4. Any discontinuity in the ST measurements in the original data due to high noise are removed in the reconstructed data. 

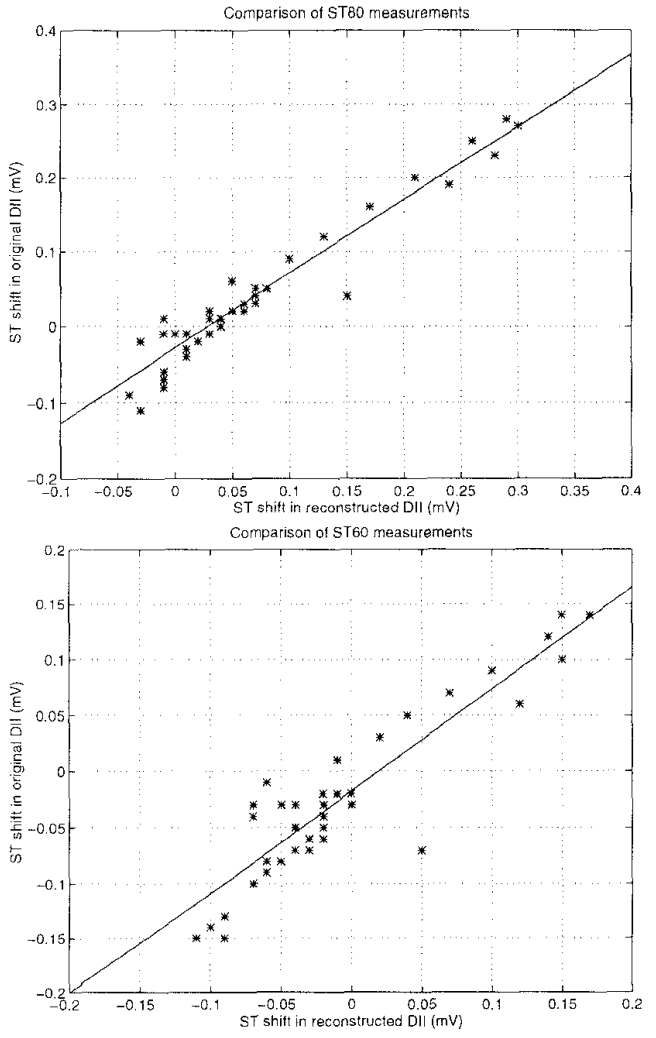

Figure 3: ST measurements from abnormal ECG

\section{Conclusion}

A new approach for online noise elimination in exercise ECG signals is described. It is based on the redundancy in standard 12 lead ECG signals. It is observed that signal components can be represented in 2 dimensional space with preserving arryhthmic morphologies. Original signals can be reconstructed without noise and totally lost chamnels can be constructed providing a non-stop exercise test.

This algorithm improves data compression by providing noise-free ECG signals which can be compressed more efficiently. The most important improvement is the information compression. If the analysis of ECG is done on the decomposed signal set, which is guaranteed to contain all the information, then keeping this reduced dimensional signal set would be enough, which provides a compression of 1 to 4 . As a first step, QRS detection on decomposed signals was done by Çağlar [5]. Further research is needed on arryhthmia detection and classification on these low dimensional orthogonal ECG signals.
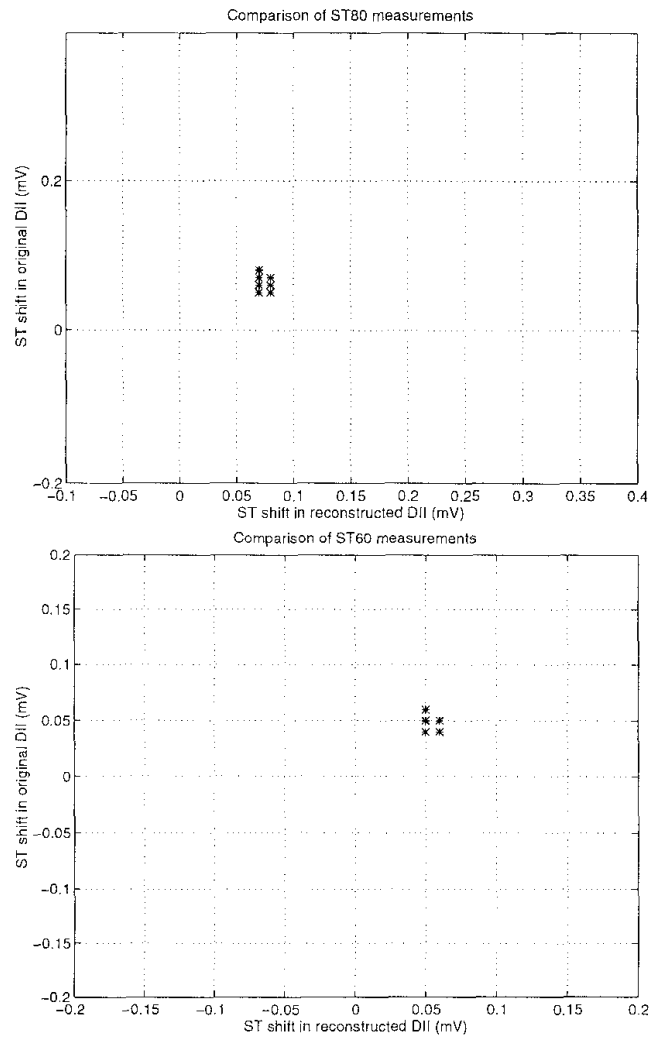

Figure 4: ST measurements from normal ECG

\section{References}

[1] Afonso V.X., Tompkins W.J., Nguyen T.Q., Michler K., Luo S., "Comparing stress ECG enhancement algorithms," IEEE EMB Magazine, vol. 15, No. 3,pp. 37-44, May/June 1996.

[2] Acar B., "Singular value decomposition based online ECG signal orthogonalization," To be submitted as an M.S. Thesis to Bilkent University, Ankara, Turkey, September 1996.

[3] Vanderschoot J., et al. "Two methods for optimal MECG elimination and FECG detection from skin elctrode signals," IEEE Trans. On Biomedical Eng., vol. BME-34, No. 3, pp. 233-242, March 1987.

[4] Golub G.H., Van Loan C.F., "Matrix Computations,", John Hopkins University Press, Baltimore, 1989.

[5] Cağlar K., "QRS detection on orthogonalized signals," To be submitted as an M.S. Thesis to Bilkent University, Ankara, Turkey, September 1996.

Address for correspondence:

Bilkent University

Eng. Fac. EE Dept.

06533 Ankara Turkey

tel/fax: $++90-312-2664307$

e-mail: buraka@ee.bilkent.edu.tr 